\section{An unusual complication of percutaneous endoscopic gastrostomy (PEG) placement in the presence of a large pharyngeal pouch}

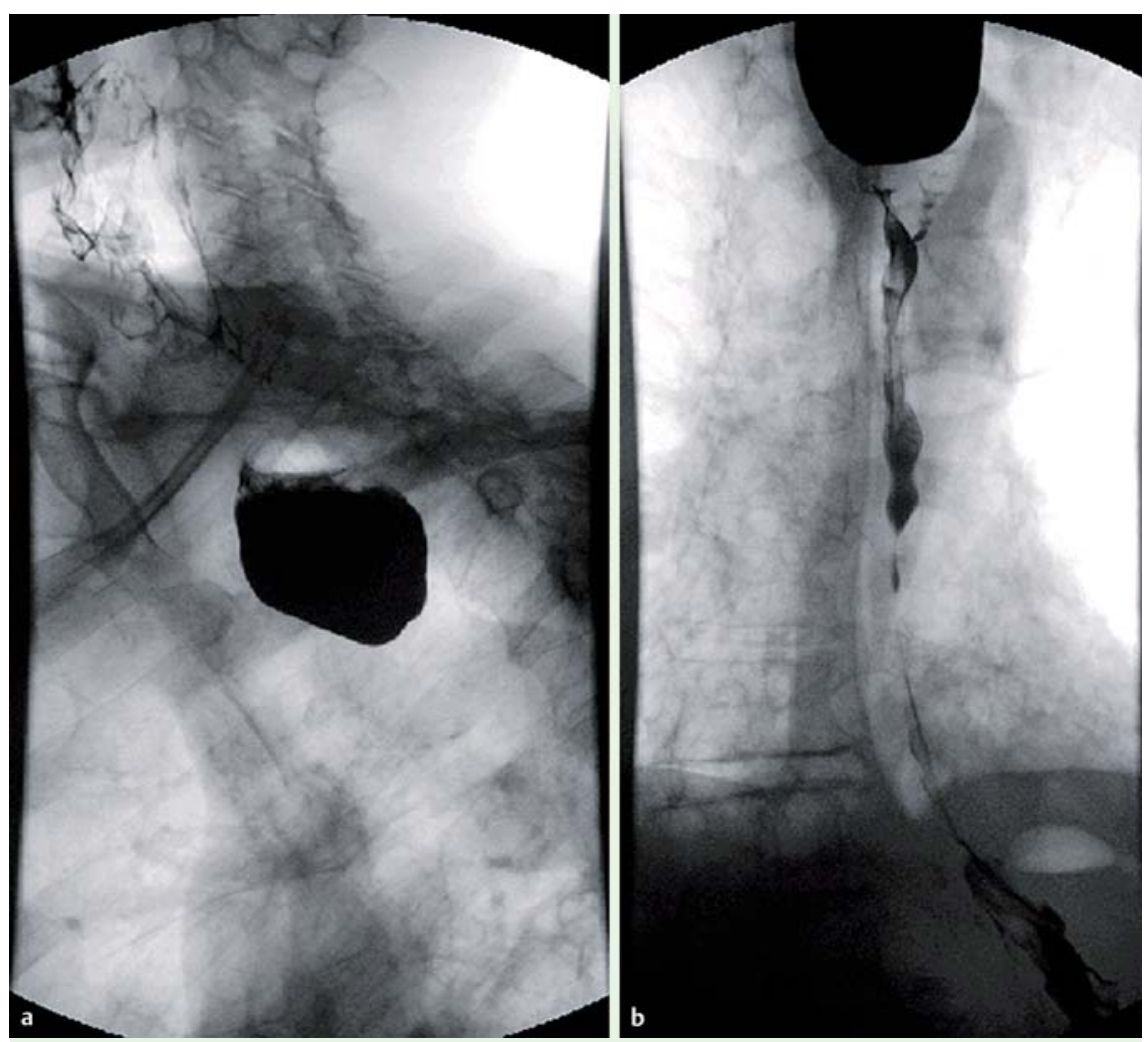

Fig. 1 Contrast swallow studies in an 80-year-old man with a known benign lower esophageal stricture and pharyngeal pouch and dysphagia. a Lateral view shows contrast in the pouch. $\mathbf{b}$ Anterior view shows contrast in the pouch and esophagus, as well as aspirated contrast in the trachea.
An 80-year-old man with a known benign lower esophageal stricture and pharyngeal pouch was admitted with a 2-week history of malaise, dysphagia, regurgitation, and weight loss. He had elected not to have the esophageal stricture dilated 3 years earlier. On this admission, the dysphagia persisted despite dilatation of the esophageal stricture, and a contrast swallow study demonstrated most of the contrast entering the pouch, with little entering the esophagus ( $\nabla$ Fig. 1 a); there was some aspiration of contrast ( $\bullet$ Fig. $\mathbf{1 b}$ ). The patient was nutritionally too frail for repair of the pharyngeal pouch $[1,2]$. Nasogastric tube insertion failed and a decision was taken to insert a percutaneous endoscopic gastrostomy (PEG) via the "pull technique" [3].

On intubation, the esophageal stricture required further dilation to allow passage of the smallest gauge PEG (9-Fr Freka-PEG, Cheshire, UK). However, on applying traction to the guiding string, resistance was obtained before the PEG had fully exited the stomach. At endoscopy, the internal bumper was found sitting flat across the opening of the pouch rather than within the esophageal lumen ( $\bullet$ Fig.2a). Downward traction on the string caused downward traction on the junction between the pouch and esophagus, instead of permitting passage of the bumper. To allow passage of the internal bumper, its inferior aspect was grasped with stentretrieval forceps ( $\mathbf{F i g . 2 b}$ ) and lifted up while traction was applied to the guiding string ( Fig. $2 \mathrm{c}-\mathrm{e}$ ).

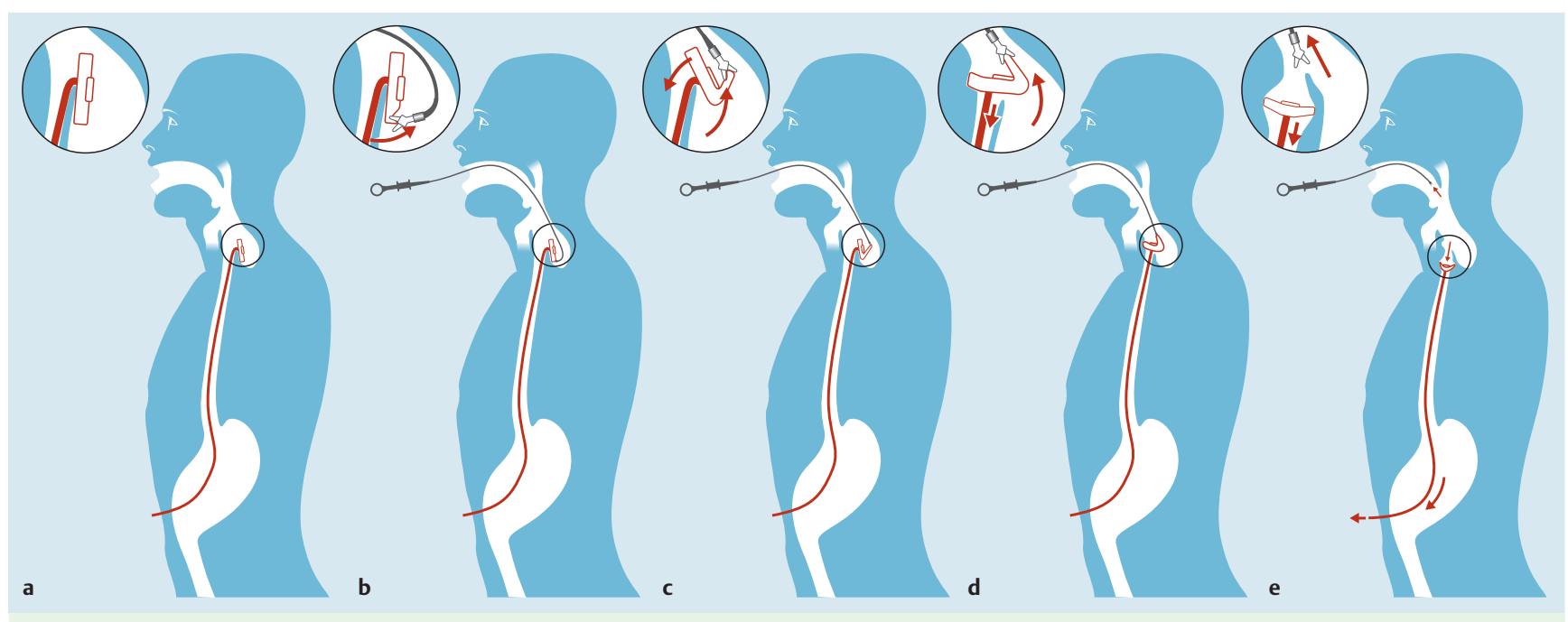

Fig. 2 a-e lllustration of the technique described in the text for percutaneous endoscopic gastrostomy (PEG) placement. 
We are unaware of such a complication being reported previously. Although the complication was resolved, the procedure was technically difficult in the confined space of the pouch and might have failed. Caution is indicated for PEG insertion in the presence of a large pharyngeal pouch, and the "push technique" [3] or a radiologically inserted gastrostomy (RIG) [4] should be considered.

Endoscopy_UCTN_Code_CPL_1AH_2AI

Competing interests: None

\section{J. Patel, A. Jenkins}

Princess Royal University Hospital, Farnborough Hospital, Orpington, Kent, United Kingdom

\section{References}

1 Zaninotto G, Narne S, Costantini $M$ et al. Tailored approach to Zenker's diverticula. Surg Endosc 2003; 17: 129-133

2 Repici A, Pagano N, Romeo F et al. Endoscopic flexible treatment of Zenker's diverticulum: a modification of the needle-knife technique. Endoscopy 2010; 42: 532-535

3 Tucker AT, Gourin CG, Ghegan MD et al. "Push" versus "pull" percutaneous endoscopic gastrostomy tube placement in patients with advanced head and neck cancer. Laryngoscope 2003; 113: 1898-1902

4 Galaski A, Peng WW, Ellis M et al. Gastrostomy tube placement by radiological versus endoscopic methods in an acute care setting: a retrospective review of frequency, indications, complications and outcomes. Can J Gastroenterol 2009; 23: 109-114

\section{Bibliography}

Dol http://dx.doi.org/ 10.1055/s-0032-1326261 Endoscopy 2013; 45: E217-E218

(c) Georg Thieme Verlag KG Stuttgart · New York ISSN 0013-726X

\section{Corresponding author}

\section{J. Patel}

Princess Royal University Hospital Farnborough Hospital

Orpington

Kent BR6 8ND

United Kingdom

Janisha.patel@doctors.org 